\title{
Crafting a Real-Time Information Aggregator for Mobile Messaging
}

\author{
Jenq-Shiou Leu \\ Department of Electronic Engineering, National Taiwan University of Science and Technology, Taipei 106, Taiwan \\ Correspondence should be addressed to Jenq-Shiou Leu, jsleu@mail.ntust.edu.tw \\ Received 7 December 2009; Revised 30 July 2010; Accepted 12 August 2010 \\ Academic Editor: Periklis Chatzimisios \\ Copyright () 2010 Jenq-Shiou Leu. This is an open access article distributed under the Creative Commons Attribution License, \\ which permits unrestricted use, distribution, and reproduction in any medium, provided the original work is properly cited. \\ Mobile messaging is evolving beyond SMS (Short Message Service) text messaging with the introduction of MMS (Multimedia \\ Messaging Service). In the past, such a scheme is used for peer-to-peer communication. Messages are generally displayed \\ on a cellular phone with a limited-sized screen. However, such a visualizing process is not suitable to broadcast real-time \\ SMS/MMS messages to people in public. To facilitate the instancy and publicity, we develop a real-time information aggregator- \\ Visualizing SMS and MMS Messages System (VSMMS)—-to realize the concept by integrating SMS and MMS messaging over \\ GSM/GPRS/UMTS onto a remote display device. The device exhibits messages on a larger display device in public. VSMMS \\ features a revolutionized variation of mass media broadcasting. In this paper, we practically illustrate how to design and implement \\ VSMMS and use a M/M/1 model to conduct a theoretical analysis about the message delay in the system queue. Meanwhile, we \\ make an empirical performance evaluation about the message transmission time over different networks.
}

\section{Introduction}

SMS [1] enables mobile subscribers to send and receive instant messages of up to 160 bytes. Some real-time information can be delivered to message receivers by SMS messages from applications, for example, delivering system failure information to the administrators [2], monitoring remote data [3]. SMS also supports many interdisciplinary applications, like Classroom Feedback Systems (CFSs) [4] to raise large-class interactivity among teachers and students. It has exploded in popularity with cell phones and other communications devices that support text messaging. A general SMS messaging system architecture includes a Short Message Service Center (SMSC), a SMS broker, and a content provider [5]. Compared with SMS, MMS [6] provides a richer message service within images, audio, text, video clips, and combinations of these. However, the screen size of cellular phone on which messages are shown is very limited. Therefore, a comprehensive displaying mechanism can enrich the visualization of mobile message communications.

Based on the above, we combined the Java Web Service, Java Secure Socket Extension, and Java Swing [7] to develop the VSMMS on a larger display device such as
PDP within a computer. Additionally, the system contains an optional interface of previewing all incoming messages by an administrator to prevent the device from displaying improper contents in public. This system can be applied to broadcast instant information sent from cellular phones onto the display device.

The rest of this paper is organized as follows. Section 2 depicts the system architecture of VSMMS and how to implement such a system, including the process flow and related system components. Section 3 presents system service analyses. Section 4 shows the comparisons between the modern messaging communication systems and VSMMS as well as some potential applications of VSMMS. Concluding remarks are finally drawn in Section 5.

\section{Architecture Design and Implementation}

2.1. System Components. VSMMS is composed of four modules as follows and the system architecture is shown in Figure 1:

(1) SMS/MMS Messages Receiver,

(2) Message Queue, 


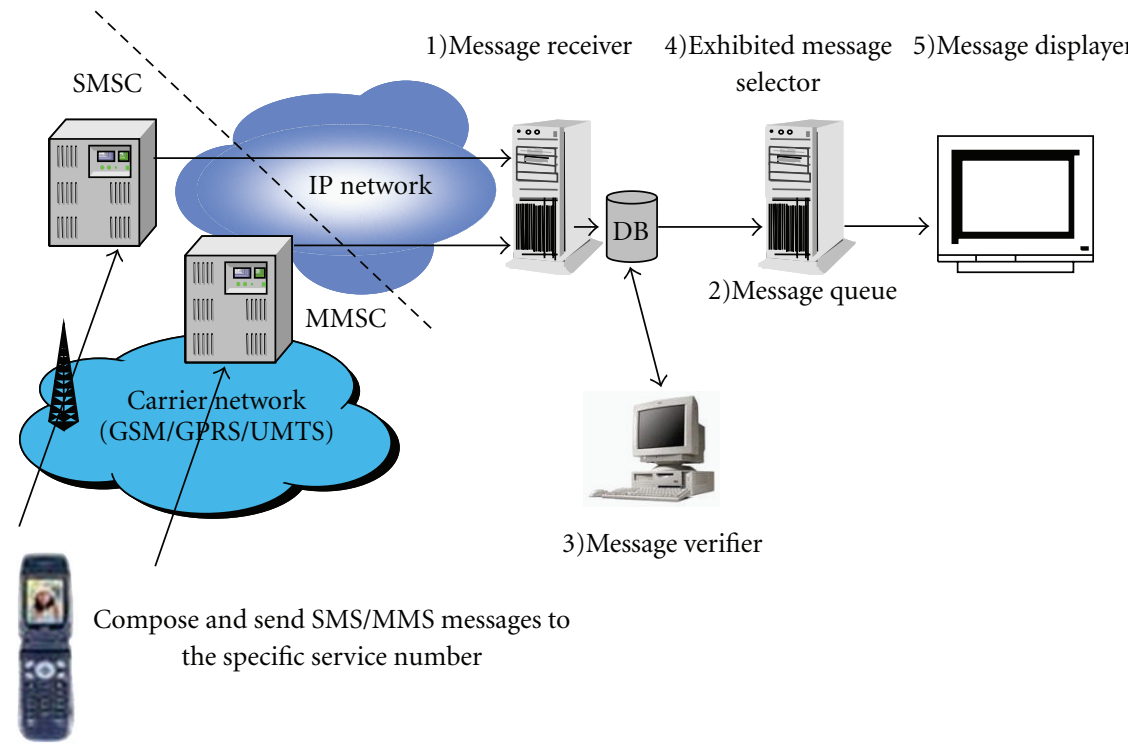

FIGURE 1: VSMMS system architecture.

(3) Message Verifier (Optional),

(4) Message Displayer,

(5) Exhibited Message Selector.

2.2. Processing Flow. Figure 2 describes the message processing logic flow.

(1) SMS messages and MMS messages are composed and sent by mobile handsets and then delivered to SMSC and MMSC, respectively.

(2) These incoming messages are forwarded to "Message Receiver" first and stored in the unverified message queue.

(3) After the administrator preview by using "Message Verifier" and then designate these messages to be approved or disapproved for display.

(4) "Exhibited Message Selector" then selects the candidate messages for "Message Displayer" to display based on different algorithms and applications.

\subsection{Detailed Descriptions for System Component}

2.3.1. SMS/MMS Message Receiver. This module is responsible for receiving all incoming messages from GSM and GPRS/UMTS networks. Two significant submodules-SMS Message Receiver and MMS Message Receiver, connect to SMSC and MMSC, respectively, to receive incoming messages. The former one links up with SMSC (SMS Centre) via SMPP [8] over IP. The latter one communicates with MMSC (MMS Centre) via MM7 interface [9] over SOAP/HTTP/IP. Detailed protocol stacks are illustrated in Figure 3. All messages targeting at the specific service ID are conveyed from SMSC/MMSC to such a receiver and put into the message queue to be further processed.

2.3.2. SMS/MMS Message Queue. Message Queue stores all incoming messages. Each entity in the queue contains the

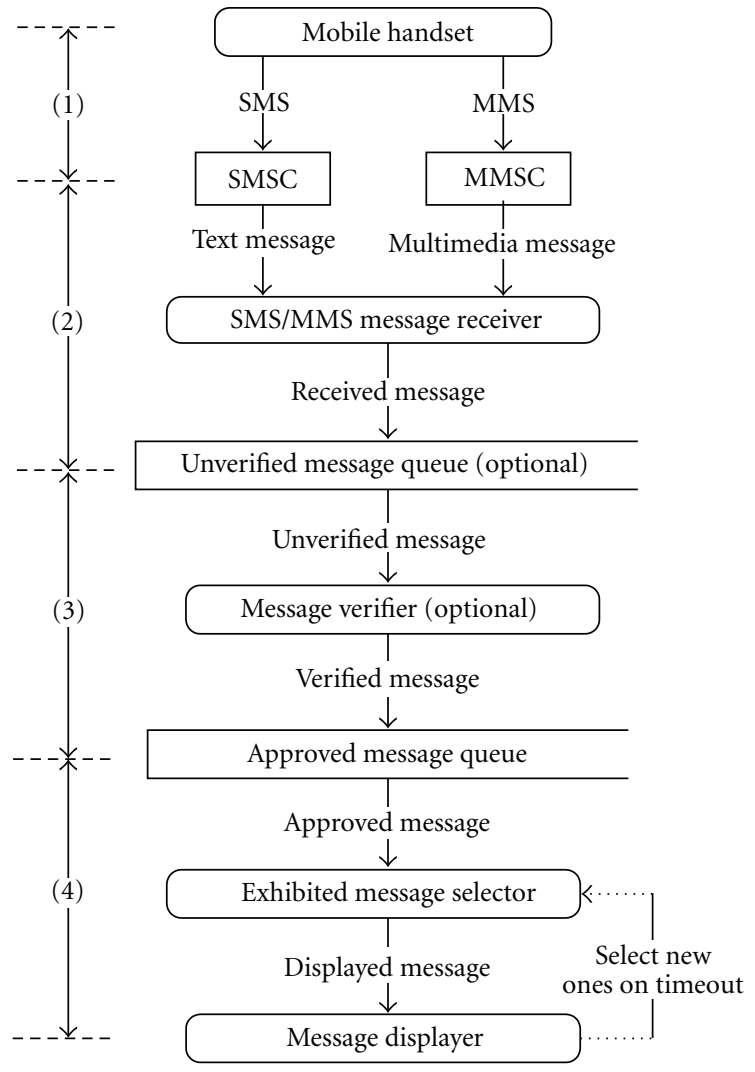

Figure 2: Message Processing Logic Flow.

generic information listed in Table 1. Such information can provide necessary parameters to the latter Exhibited Message Selector on a selection basis, for example, "MessageType", "CreatedTime", and "LastDisplayedTime" according to different scheduling mechanisms. 


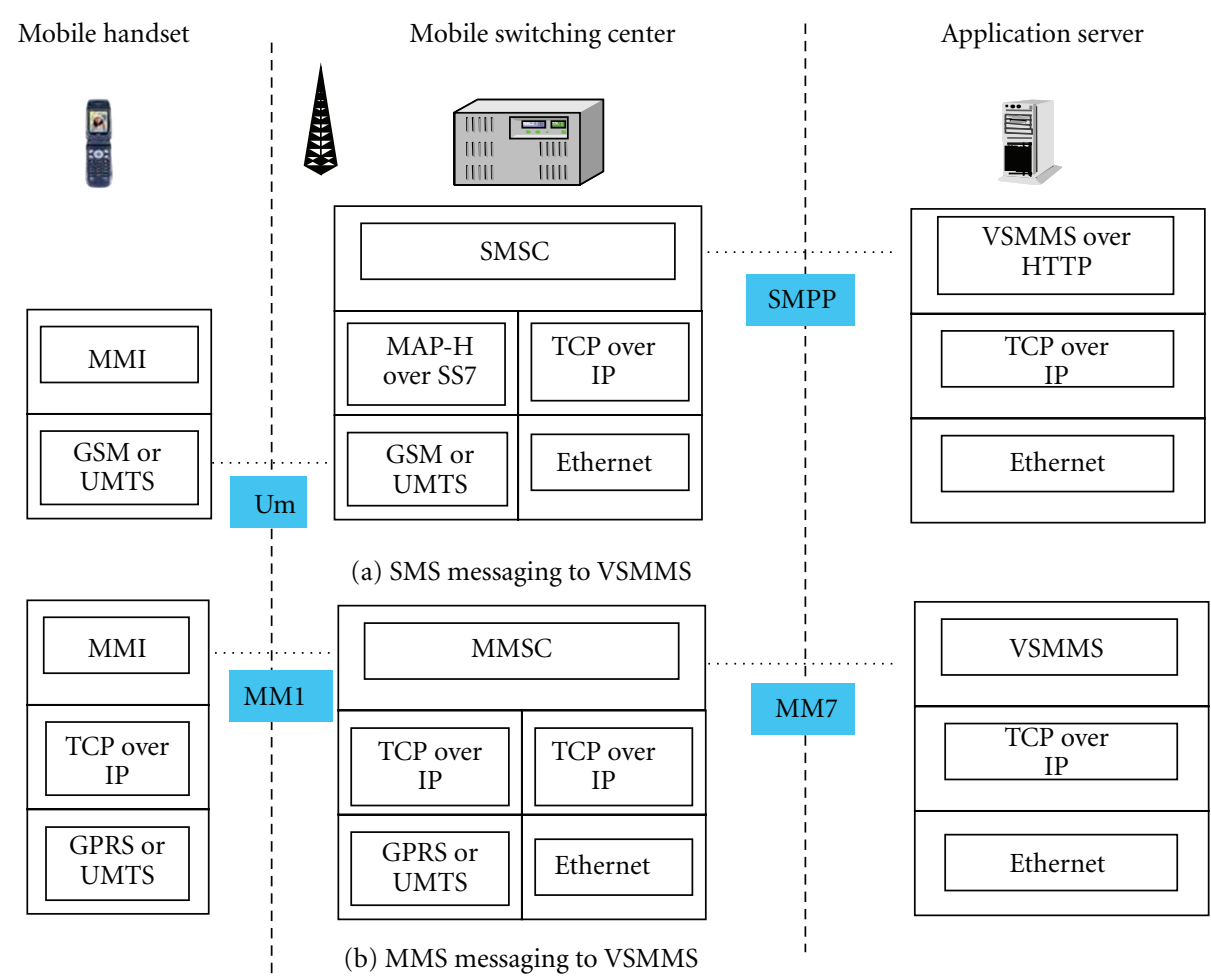

FIGURE 3: SMS/MMS messaging to VSMMS protocol stack.

TABLE 1: Attributes of messages.

\begin{tabular}{ll}
\hline Attributes & Description \\
\hline ID & Message entity ID \\
Message ID & SMS/MMS message ID \\
CreatedTime & message created time \\
Priority* & Selected to be exhibited priority \\
MSISDN & Sender's phone number \\
Text & Text part in message \\
Image & Image part in message \\
MessageType & What type the message is \\
LastExhibitedTime & Last exhibited time \\
Flag & Verification state \\
\hline
\end{tabular}

*For priority driven selection.

$* *$ If the message does not need to be verified before exhibited, the "Flag" for messages in the queue is set to be "Approved" by default.

2.3.3. Message Verifier (Optional). Since the service ID is opened for all users, a simple mechanism to administer contents in messages can avoid displaying improper contents from malicious senders. An authorized checker can utilize Message Verifier to verify all incoming messages via a webbased interface. After finding out thatthere exist some unverified messages in the system, the checker can examine all constituents in each message, including the text description or media content, to decide which message can be approved or disapproved to publish. By selecting the "Approve" or "Disapprove" radio button as shown in Figure 4, all verified messages can be submitted to the system for displaying.

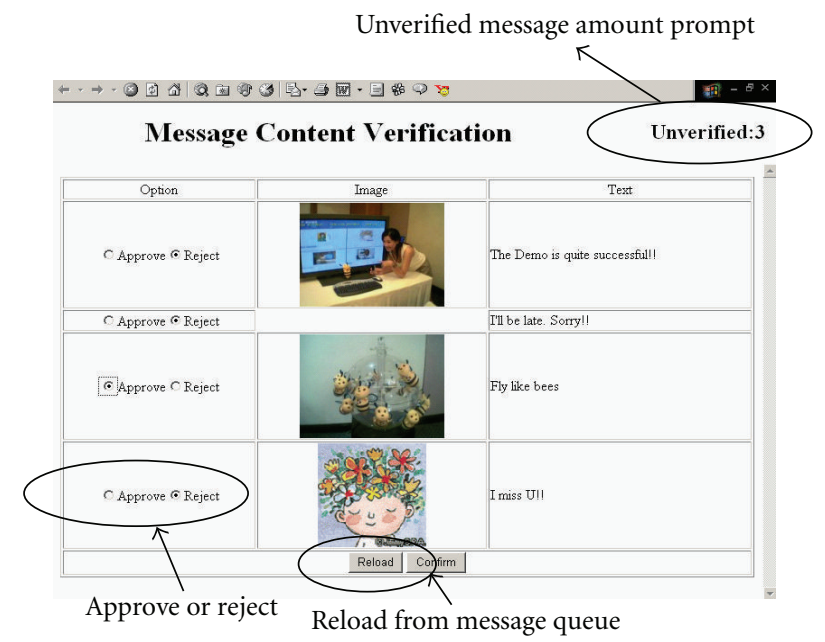

Figure 4: An optional web-based interface to preview message contents.

2.3.4. Exhibited Message Selector. Exhibited Message Selector performs a selection among approved messages for Message Displayer. Each region in the layout of Message Displayer has an independent message assigned by Exhibited Message Selector. Such a component can be implemented by different scheduling algorithms, such as First-Come-First-Served (FCFS), Round-Robin manner, or priority-driven manner by referring to necessary fields, such as "CreatedTime" and "Priority". In order to conduct a selection with fairness for all approved messages for exhibition, we exploit a hybrid 
// Select $K$ messages in the approved message queue as requested

(1) Count all fresh messages, which are not yet exhibited, (with NULL value in "LastExhibitedTime" field) and set such the said count to $N$

(2) If $N>K$

Then

2.1 Designate the preceding $K$ messages among these $N$ messages by referring to "CreatedTime" as selected ones Else

2.2 Designate these $N$ messages as selected ones

2.3 Designate the preceding $K-N$ messages among old messages (with non-NULL value in "LastExhistedTime" field) by referring to "LastExhibitedTime" as selected ones End IF

(3) Update "LastExhibitedTime" values for these $K$ .messages as the current time

(4) Return these $K$ messages IDs

Algorithm 1: Algorithm of FCFS-based selection with LRU re-exhibition.

way of the FCFS mechanism for fresh messages and Least Recent Used (LRU) mechanism for old messages by referring to "LastExhibitedTime" as an aging factor for re-exhibition. The detailed scheme is depicted in Algorithm 1.

2.3.5. Message Displayer. This module is used to exhibit approved message contents, which are selected by Exhibited Message Selector, in the Approved Message Queue. The physical layout may depend on different applications and comprise several independent regions. Figure 5 illustrates a sample display layout. The top area is a marquee, which exhibits text message contents. The bottom area consists of four regions. MMS message contents are displayed concurrently in these four regions which include an image part and a text one accordingly. These regions can have their own display durations determined by Message Displayer. Additionally, these independent regions always ask for a new message content to display toward Exhibited Message Selector when the corresponding display durations are expired. Figure 6 shows VSMMS implemented on a computer-embedded PDP.

\section{System Service Analysis}

3.1. Message Delay Analysis. We analyze the message delay in the message queue of VSMMS by a M/M/1 queuing conceptual model shown in Figure 7. The assumptions and parameters exploited in the model are illustrated below.

(1) The new SMS and MMS message arrival into the approved message queue of VSMMS SMS/MMS are Poisson distributions with rates $\lambda_{S M S}$ and $\lambda_{M M S}$, respectively. The total message arrival rate is $\lambda=$ $\lambda_{S M S}+\lambda_{M M S}$.
(2) The exhibition time of a message is assumed to be exponentially distributed with mean $1 / \mu$. All stored messages are displayed according to FIFO scheduling.

(3) The message queue in VSMMS is a finite storage with a capacity of $M$ messages.

Performance analysis of the VSMMS message queue can be induced by describing the queue as a Markov chain. Figure 8 shows the transitions among different states where the state $i$ indicates that there are $i$ messages in the queue.

Let $P_{i}$ denote the steady-state probability of being in the state $i$. Using balance equations, we can get the following result:

$$
P_{i}=\prod_{j=1}^{i}\left(\frac{\lambda}{\mu}\right) P_{0}=P_{0}\left(\frac{\lambda}{\mu}\right)^{i}, \quad 1 \leq i \leq M+1,
$$

where $P_{0}$ can be as $\sum_{i=0}^{M+1} P_{i}=1$.

Let $a_{i}$ denotes the probability of having $i$ messages in the queue just before a message arrives and gets accepted by the VSMMS. Such a message never sees the queue in the state $M+1$, that is, blocking state. $a_{i}$ can be shown by the following result $[10,11]$.

$$
a_{i}=\frac{P_{i}}{1-P_{M+1}} .
$$

The message delay $(W)$ is defined as the time between the acceptance of a message in VSMMS and the time the message starts to be transmitted. Two notations $F_{z}(t)$ and $f_{z}(t)$ are used for the following analyses. $F_{z}(t)$ and $f_{z}(t)$ denote the distribution and density function of a random variable $z$, where

$$
f_{z}(t)=\frac{d}{d x} F_{z}(t)
$$




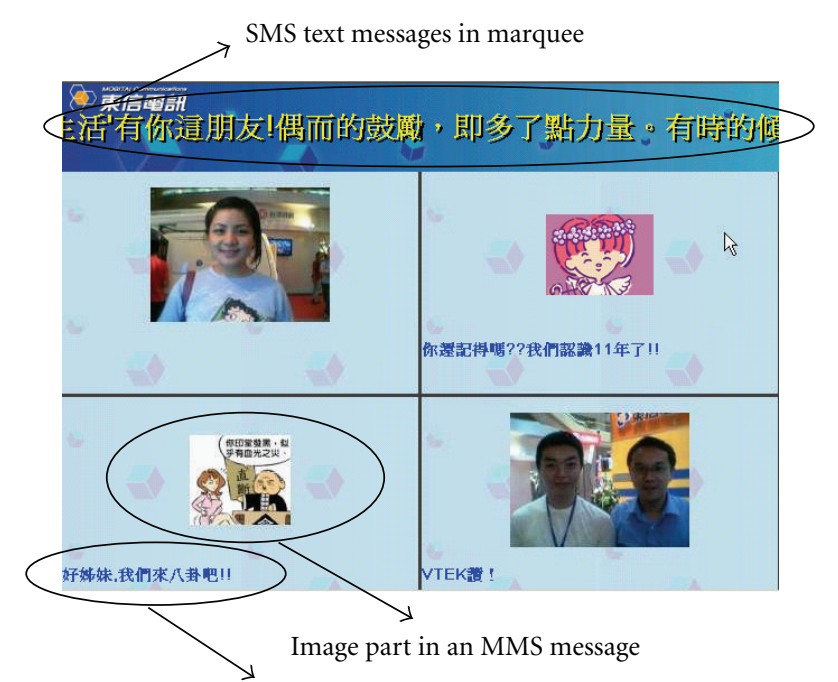

Text part in an MMS message

Figure 5: Layout of message displayer.

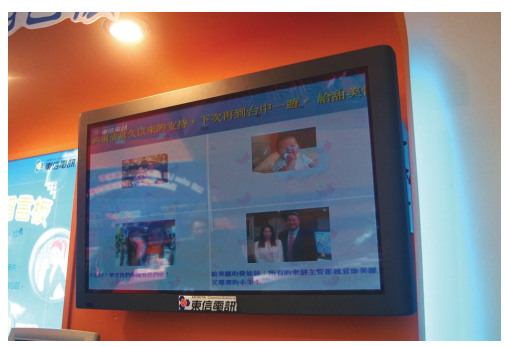

FIGURE 6: VSMMS on a computer-embedded PDP.

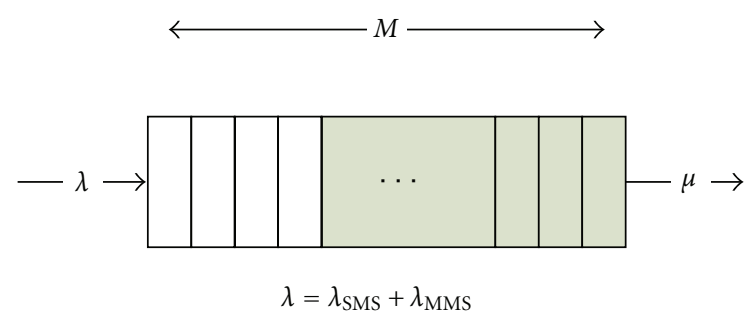

Figure 7: A queuing model for the VSMMS message queue.

Assume that a message arrives to the queue when there are $i$ messages in the queue (i.e., the queue is in the state $i$ ). Let $m_{i}$ denote this message. If $1 \leq i \leq M+1$, then $m_{i}$ is accepted and the queue state would change into $i+1$. If $i=0$, then $m_{i}$ would be immediately processed, otherwise it must wait in the queue till the $i$ message has been processed. Suppose the queue is consisted of these $i$ messages which are ahead of $m_{i}$ at some moment. Let $t_{i}$ denote the time required for the message population to decrease from $i$ to $i-1(1 \leq i \leq M)$. Then, $t_{i}$ is exponentially distributed with the rate parameter $\mu$, and we can obtain

$$
f_{t_{i}}(t)=\mu e^{-\mu t}
$$

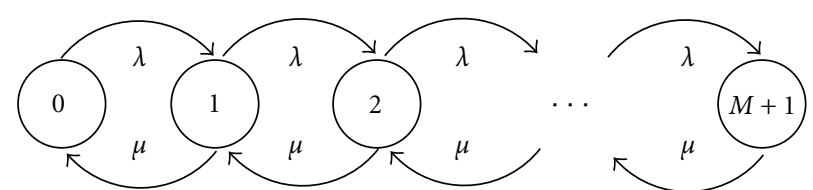

Figure 8: An M/M/1 Markov chain representation for the VSMMS message queue.

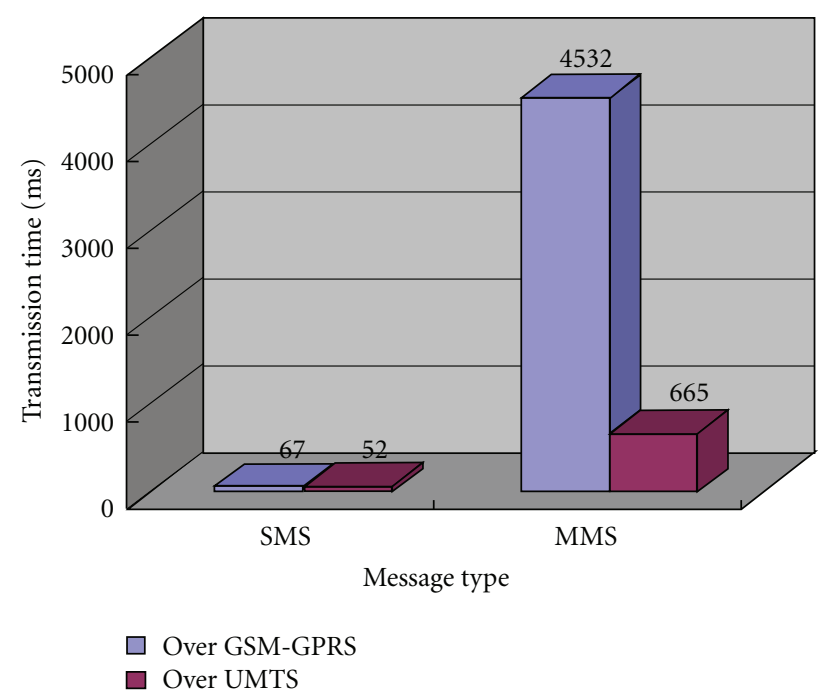

FIGURE 9: Average transmission time of SMS/MMS messaging over GSM/GPRS/UMTS.

Let $T_{i}$ denote the message delay of $m_{i}$, that is, the amount of time $m_{i}$ must wait before its transmission starts. Then we can get

$$
T_{i}=t_{1}+t_{2}+\cdots+t_{i}=T_{i-1}+t_{i}, \quad \text { for } i \geq 1, T_{0}=0 .
$$

The mean message delay is therefore given by

$$
E\left[T_{i}\right]=\sum_{j=1}^{i} E\left[t_{j}\right]=\sum_{j=1}^{i} \frac{1}{\mu}=\frac{i}{\mu} .
$$

3.2. Transmission Time Analysis. VSMMS offers an instant way to gather and broadcast instant messages from anywhere under cellular network coverage, for example, $53.6 \mathrm{kbps}$ over GPRS or $384 \mathrm{kbps}$ over UMTS for a limited radio resource. The consumed time of message transmission may be an attractive issue. Normally SMS messages fly over SS7 control channels, the transmission time for SMS messages is far below the one consumed by transmitting MMS messages. (Figure 9 shows the average transmission time of SMS/MMS messaging over GSM/GPRS/UMTS while delivering 102-byte SMS messages and 24-kbyte MMS message to VSMMS.) Hence, the focusing scenario is to compose and deliver MMS messages to VSMMS. The consumed time for such a scenario is shown by

$$
\left(\frac{\text { Size }_{\mathrm{MMS}}}{\mathrm{TR}_{\mathrm{GPRS} / \mathrm{UMTS}} \mathrm{UL}}+\frac{\text { Size }_{\mathrm{MMS}}}{\mathrm{TR}_{\mathrm{GPRS} / \mathrm{UMTS} \text { DL }}}\right) \times 8 \text { seconds, }
$$


TABLE 2: Comparisons of the contemporary messaging communication systems and VSMMS.

\begin{tabular}{llll}
\hline & Mobile messaging system & VSMMS & Digital broadcasting system \\
\hline $\begin{array}{l}\text { Communication model } \\
\text { Mobility for message creator }\end{array}$ & 1 to 1 & 1 to Many in One Centralized Receiver & $\begin{array}{l}\text { 1 to Many with scattered } \\
\text { receivers }\end{array}$ \\
$\begin{array}{l}\text { Immediateness of message } \\
\text { delivery }\end{array}$ & High & High & Low \\
$\begin{array}{l}\text { Scale for message receiving } \\
\text { Carrier network }\end{array}$ & $\begin{array}{l}\text { Small } \\
\text { Cellular network } \\
\text { (GSM/GPRS/UMTS) }\end{array}$ & $\begin{array}{l}\text { High } \\
\text { to any all-IP networks) }\end{array}$ & Low \\
Message capacity & $\begin{array}{l}\text { Low (limited by handset } \\
\text { form factor) }\end{array}$ & High (System-based, extendable) & $\begin{array}{l}\text { Ligh (System-based, } \\
\text { extendable) }\end{array}$ \\
\hline
\end{tabular}

where Size $_{\text {MMS }}$ denotes the MMS message size in kbyte;

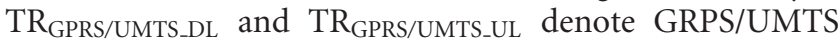
transmission rates in kbits per second for the downlink and uplink, respectively.

For example, the size of an MMS message is $26.8 \mathrm{kbytes}$ and the GPRS transmission rate is $53.6 \mathrm{kbps}$. The total consumed time from posting to being received by VSMMS is $4 \sim 5$ seconds.

\section{Analytical Comparison and Potential Applications}

Compared to modern messaging communication systems, such as Mobile Messaging System and Digital Broadcasting System [12], VSMMS features one-to-many mediumsized broadcasting on a centralized display device with high mobility and instancy support via cellular networks. Furthermore, VSMMS can be easily extended and adapted to the future all-IP networks. The major differences between the contemporary messaging communication systems and VSMMS are depicted in Table 2.

VSMMS can be one of the following variations but not limited.

4.1. Real-Time Traffic Information Sharing. Traffic jam is getting worse for lack of real-time traffic information. Drivers can use MMS-enabled handsets within a digital cam to capture and advertise the visible traffic condition in public, instead of only oral reporting.

4.2. Home Appliance within a Family Bulletin Board. A dedicated channel in a traditional TV allocated for unfolding SMS/MMS messages enables people to communicate with one another like a family bulletin board.

4.3. Dynamic Poster Framework. To substitute for the traditional public bulletin board, a multimedia display terminal with wireless instead of wired communication capability enables the flexibility of dynamic advertisement or announcement in a public area, especially for locationoriented affairs like community information, weather information.
4.4. Real-Time Visualization for Televoting Statistics. Televoting service can establish direct connections with radiostations listeners, TV viewers, and readers of newspapers and magazines. Such a visualization process can make mobile voting mechanism more evident.

\section{Conclusion}

The prototype system presented in this paper has been successfully implemented and demonstrated. We have realized an extended idea to visualize instant SMS/MMS messages openly on a larger device, instead of a limited-sized screen on personal cellular phones in the past. Such an implementation also improves the traditional peer-to-peer mobile communication. Users compose SMS or MMS messages within real-time information by handset and send them to a specific service ID via SMSC/MMSC. Afterward all incoming messages are processed by VSMMS and shown on a remote display device. VSMMS is suitable to be deployed for realtime information sharing in public. To avoid displaying improper contents in public, the system includes an optional web-based interface to help the administrator verify the incoming contents. VSMMS features a novel messaging communication scheme for mass media broadcasting.

\section{References}

[1] 3GPP TS 23.040, "Technical realization of the short message service (SMS), release 5," v5.2.0, 2001.

[2] M. Ghasemzadeh and V. A. Foroushani, "Remote management of computer networks by short message service," in Proceedings of the International Conference on Computer and Communication Engineering (ICCCE '08), pp. 300-305, May 2008.

[3] H. Yan and H. Pan, "Remote data monitoring system design based on GSM short message service," in Proceedings of IEEE International Symposium on Industrial Electronics, pp. 364$367,2009$.

[4] E. Scornavacca, S. Huff, and S. Marshall, "Mobile phones in the classroom: if you can't beat them, join them," Communications of the ACM, vol. 52, no. 4, pp. 142-146, 2009.

[5] J. Brown, B. Shipman, and R. Vetter, "SMS: the short message service," Computer, vol. 40, no. 12, pp. 106-110, 2007.

[6] 3GPP TS 22.140, "Multimedia messaging service (MMS); stage 1; release 6," v6.6.0, June 2004. 
[7] For Java Developers, 2010, http://www.oracle.com/technetwork/java/index.html.

[8] SMPP Forum, "Short Message Peer-to-Peer Protocol Specification v5.0," 2010, http://www.smsforum.net/.

[9] 3GPP TS 23.140, "Multimedia messaging service (MMS); functional description; stage 2; release 6," v6.6.0, June 2004.

[10] D. Gross, et al., Fundamentals of Queueing Theory, John Wiley \& Sons, New York, NY, USA, 4th edition, 2008.

[11] M. Ghaderi and S. Keshav, "Multimedia messaging service: system description and performance analysis," in Proceedings of the 1st International Conference on Wireless Internet (WICON '05), pp. 198-205, July 2005.

[12] ETSI and EN 300 744, "Digital video broadcasting (DVB): framing structure, channel coding and modulation for digital terrestrial television," vol. 1.5.1, pp. 9-39, November 2004. 

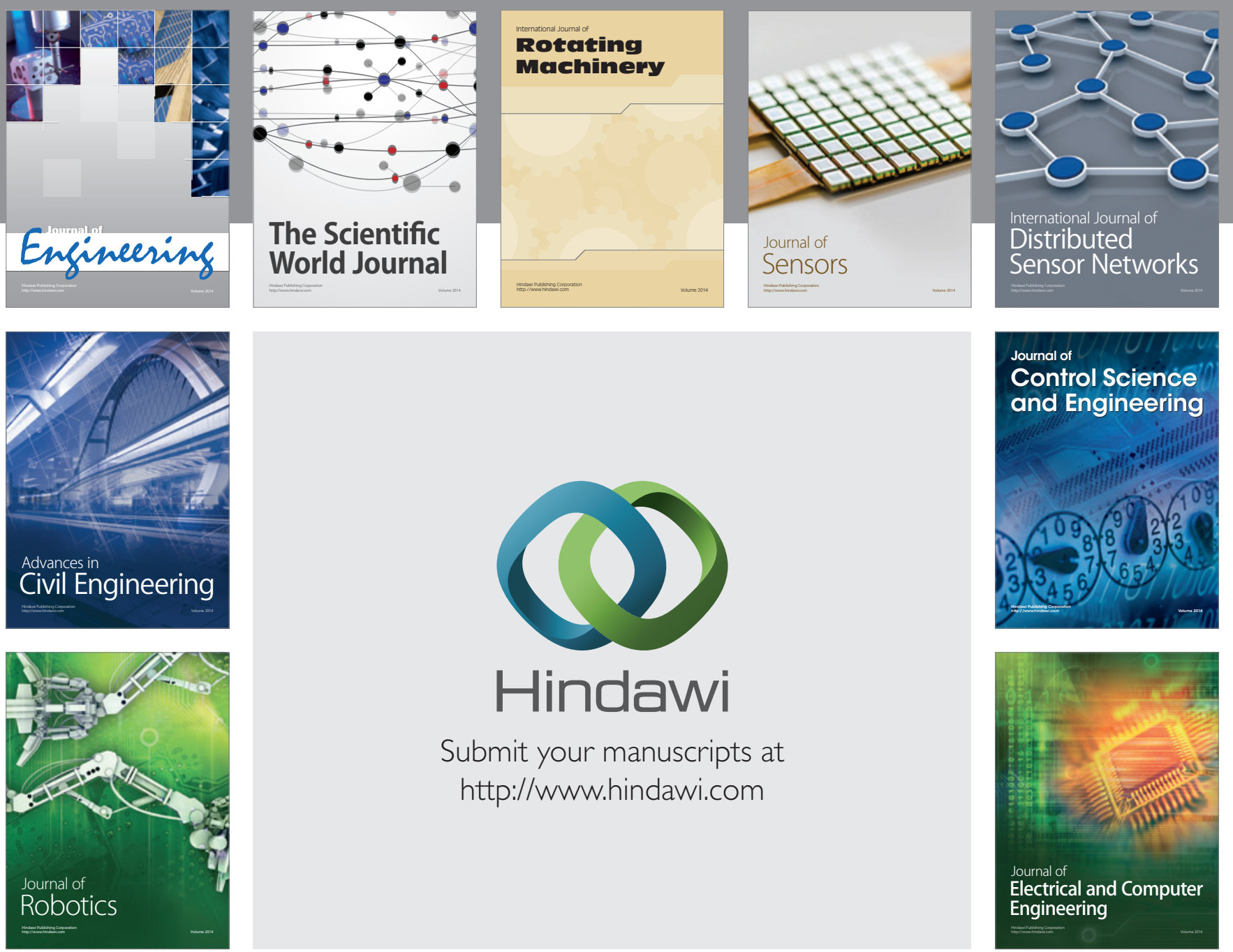

Submit your manuscripts at

http://www.hindawi.com
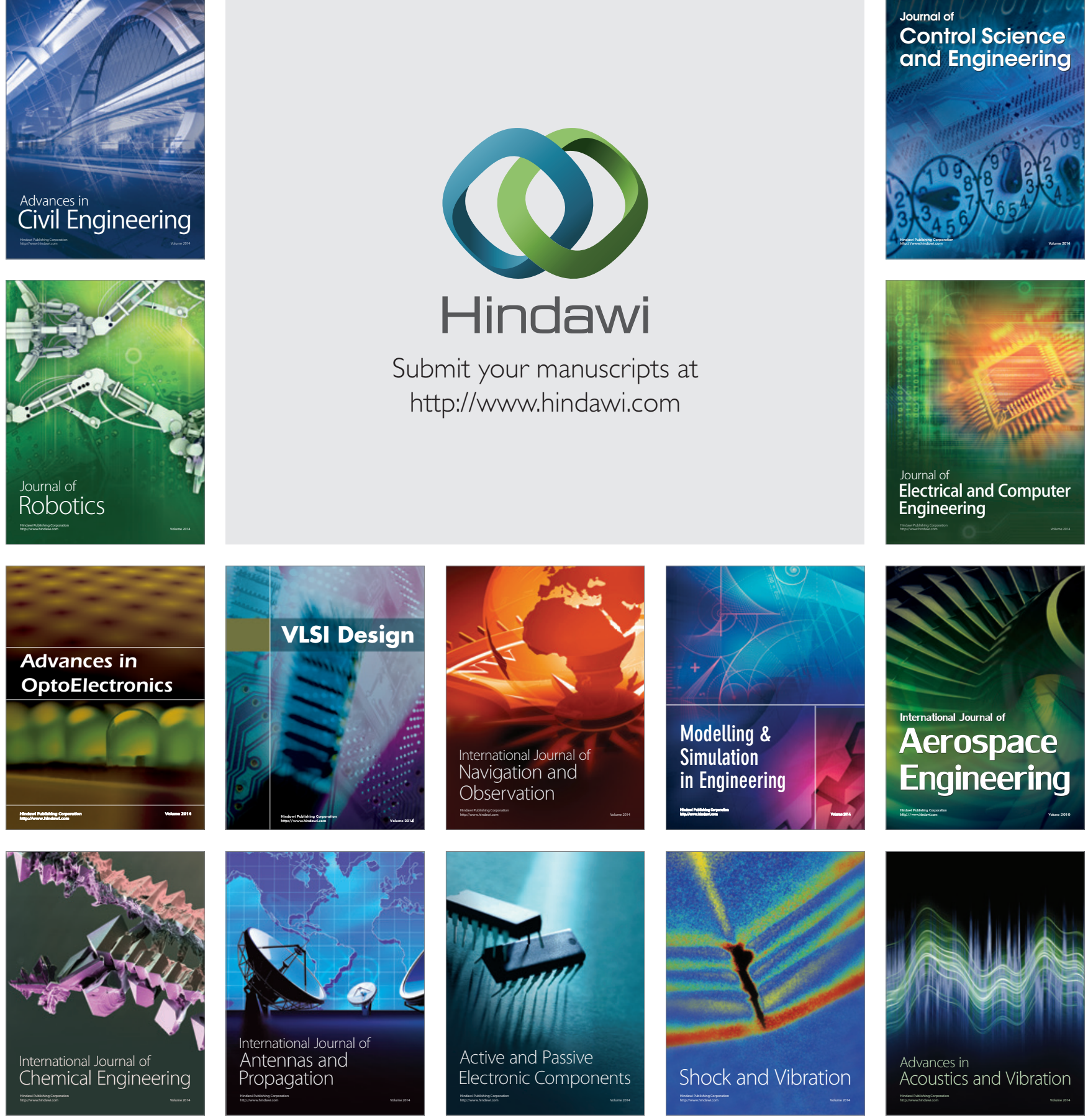